\section{Junge Ärzte wollen forschen - gegen den Attraktivitätsverlust der akademischen Forschung}

Eine hochwertige Patientenversorgung benötigt medizinischen Fortschritt und wissenschaftlich versierte Ärzte. Hierfür bedarf es einer hochwertigen Grundlagen- und klinischer Forschung. Das Bündnis JUNGE ÄRZTE (BJÄ) fordert eine Verbesserung der Rahmenbedingungen, um den wissenschaftlichen Nachwuchsmangel abzuwenden und so die Attraktivität und Leistungsfähigkeit der akademischen und evidenzbasierten Medizin in

Deutschland in einem weltweit zunehmend kompetitiven Umfeld zu sichern.
Forschende Ärzte sind mit deutlichen Erschwernissen konfrontiert: Meist verlängert sich durch das wissenschaftliche Arbeiten die Facharztweiterbildung und führt zu einer zeitlichen und inhaltlichen Zusatzbelastung auf Kosten der WorkLife-Balance. Zudem bestehen unnötige bürokratische Hürden. Eine fehlende Wertschätzung und finanzielle Ungleichheit in nicht klinischen Angestelltenverhältnissen machen die medizinische Forschung als Arzt noch unattraktiver. Daher entscheiden sich immer mehr junge Ärzte gegen eine akademische Laufbahn oder suchen Alternativen im Ausland, wo Klinik und Forschung besser vereinbar sind.

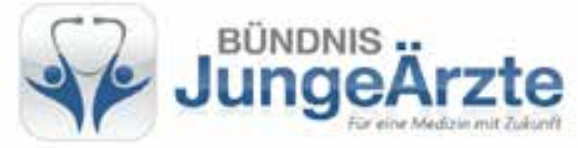

Die Folge ist ein Nachwuchsmangel von wissenschaftlich tätigen Ärzten in Deutschland.

Diese Entwicklung hält das Bündnis JUNGE ÄRZTE für sehr bedrohlich. Seit jeher war die medizinische Forschung die unabdingbare Basis einer qualitativ hochwertigen Patientenversorgung. Das BJÄ fordert eine Stärkung des wissenschaftlichen Nachwuchses und ein Ende der „Forschung in der Freizeit“.

Das Bündnis besteht auf die Möglichkeit einer strukturierten Integration von Forschungsinhalten in die Facharztweiterbil- 
dung, um die akademische Medizin für junge Ärzte attraktiv zu gestalten. Nur durch eine strukturierte Weiterbildung des wissenschaftlichen Nachwuchses kann eine hochwertige medizinische Forschung und Patientenversorgung langfristig sichergestellt werden.

Das Bündnis JUNGE ÄRZTE fordert von - den Wissenschaftsministerien

> Die Einrichtung weiterer Forschungsstellen für wissenschaftlich interessierte junge Ärzte an Unikliniken bzw. medizinischen Fakultäten.

- Die äquivalente Bezahlung von klinisch und wissenschaftlich tätigen Ärzten. Es darf keine finanzielle Benachteiligung mehr von wissenschaftlich tätigen Ärzten geben!

- der Gesundheitspolitik

- Eine adäquate finanzielle Berücksichtigung der klinischen Sonderaufgaben der forschenden und lehrenden Einrichtungen, damit kein Bedarf mehr besteht, für die Forschung gedachte Mittel zweckentfremdet einzusetzen.

- Kliniken, die Sonderaufgaben im Bereich von Forschung und Wissenschaft sowie Aus- und Weiterbildung einnehmen, muss durch eine adäquate Abbildung und Bewertung ihrer Leistungen insbesondere im DRG-Katalog ein ausgeglichener Haushalt ermöglicht werden. Der chronischen Unterfinanzierung der akademischen Krankenhäuser muss entgegengewirkt werden!

$\checkmark$ den Geschäftsführungen und Klinikdirektoren

- Gezielte strukturelle und personelle Investitionen im Forschungsbereich.

> Prinzipiell keine Quersubvention der Patientenversorgung durch Drittmittel durchzuführen.

- Forschung ist eine Kernaufgabe der Unikliniken und muss auch in finanziell knappen Zeiten vorrangig Berücksichtigung finden. Der Trend zur Ausrichtung des Klinikmanagements nach ökonomischen Gesichtspunkten darf nicht zu einer Marginalisierung von Wissenschaft und Forschung an diesen Kliniken führen!

- der Bundesärztekammer und den Landesärztekammern

> Eine flächendeckende Anerkennung von Forschungszeiten (Grundlagenforschung und klinische Forschung) als fakultativen Inhalt der Facharzt- weiterbildung. Konkret sollen bis zu 12 Monate wissenschaftliche Tätigkeit als Weiterbildungszeit anerkannt werden können.

Weiterhin fordern wir die Abschaffung bürokratischer Hürden in der Forschung. Ziel sollte es sein, dass kein Antragsformular mehr als 3 Seiten umfasst und nicht mehr als 10\% der Arbeitszeit für bürokratische Aufgaben verwendet werden müssen!

\section{Epilog}

Wissenschaftlicher Fortschritt ist die Grundlage einer stetigen Verbesserung der Patientenversorgung. Als junge Ärzte möchten wir eine hochwertige Forschung in Deutschland langfristig sicherstellen. Hierzu brauchen wir als akademischer Nachwuchs eine realistische Perspektive und adäquate Rahmenbedingungen.

Das Bündnis JUNGE ÄRZTE fordert deshalb die Verantwortlichen dringend dazu auf, die oben genannten Fehlentwicklungen $\mathrm{zu}$ thematisieren und diesen entgegenzuwirken, um eine hochwertige Forschung und Patientenversorgung langfristig nachhaltig zu gewährleisten.

Berlin, den 10.2.2015

Dr. Gerhard Achatz - Perspektivforum Junge Chirurgie der Deutschen Gesellschaft für Chirurgie e.V.

Dr. Stephanie Anetsberger \& Dr. Jürgen Konczalla - Deutsche Gesellschaft für Neurochirurgie e.V.

Dr. Hannah Arnold - GeSRU (German Society of Residents in Urology) e.V.

Dr. Diane Bitzinger - Deutsche Gesellschaft für Anästhesiologie und Intensivmedizin e.V.

Dr. Christoph Eckert - Berufsverband der Augenärzte Deutschland e.V.

\section{Dr. Ulrike Gaiser - Deutsche Gesellschaft für Kinder- und Jugendmedizin e.V.}

Dr. Katja Hüper \& Dr. Perla Seyfer - Deutsche Röntgengesellschaft e.V.
Dr. Matthias Krüger - Berufsverband der Deutschen Chirurgen e.V.

Dr. Berend Malchow - Deutsche Gesellschaft für Psychiatrie und Psychotherapie, Psychosomatik und Nervenheilkunde e.V.

Dr. Alexis Müller-Marbach - Deutsche Gesellschaft für Innere Medizin e.V.

Dr. Anna-Katharina Doepfer \& Dr. Matthias Münzberg - Deutsche Gesellschaft für Orthopädie und Unfallchirurgie e.V.

\section{Dr. Christiana Ossig - Deutsche Gesell-} schaft für Neurologie e.V.

\section{Kourosh Roushan - Deutsche Gesellschaft für Hals-Nasen-Ohren-Heilkunde, Kopf- und Hals-Chirurgie e.V.}

\section{Dr. Katrin Schierle - Berufsverband Deut- scher Pathologen e.V.}

Dr. Sarah Schott E' Dr. Johannes Lermann Deutsche Gesellschaft für Gynäkologie und Geburtshilfe e.V.

\section{Dr. Kevin Schulte - Berufsverband Deut- scher Internisten e.V.}

Bündnis JUNGE ÄRZTE ist ein Zusammenschluss der Vertreter der jungen Ärzte (Assistenzärzte und junge Fachärzte) folgender Verbände und Fachgesellschaften: Berufsverband der Deutschen Chirurgen e.V.(BDC), Berufsverband Deutscher Internisten e.V. (BDI), Berufsverband Deutscher Pathologen e.V. (BDP), Berufsverband der Augenärzte Deutschlands e.V. (BVA), Berufsverband der Deutschen Dermatologen e.V.(BVDD), Deutsche Gesellschaft für Anästhesiologie und Intensivmedizin e.V. (DGAI), Deutsche Gesellschaft für Innere Medizin e.V. (DGIM), Deutsche Gesellschaft für Neurologie e.V. (DGN), Deutsche Gesellschaft für Psychiatrie, Psychotherapie, Psychosomatik und Nervenheilkunde e.V. (DGPPN), Deutsche Gesellschaft für Kinder- und Jugendmedizin e.V.(DGKJ), Deutsche Gesellschaft für Orthopädie und Unfallchirurgie e.V. (DGOU), Deutsche Gesellschaft für Gynäkologie und Geburtshilfe e.V. (DGGG), Deutsche Gesellschaft für Neurochirurgie e.V.(DGNC), Deutsche Gesellschaft für Hals-Nasen-OhrenHeilkunde, Kopf- und Hals-Chirurgie e.V. (DGHNO), Deutsche Röntgengesellschaft e.V. (DRG), German Society of Residents in Urology (GesRU). Ziel und Aufgabe des Bündnisses ist es, die Patientenversorgung nach modernen und ethischen Gesichtspunkten zu verbessern und Berufsbedingungen für eine Medizin der Zukunft zu gestalten. Um dies gemeinsam zu erreichen fand im Dezember 2013 der Zusammenschluss in Berlin statt. Adresse: Bündnis JUNGE ÄRZTE c/o Berufsverband Deutscher Internisten e.V. Postfach 156665005 Wiesbaden

Bündnis JUNGE ÄRZTE

Ansprechpartnerin:

Ursula-Anne Ochel

Telefon: 030-32304270

Mobil: 0171-3224346

E-Mail:

Buendnis_JUNGEAERZTE.Presse@t-online.de Web: www.jungeärzte.de 